\title{
miR-153 regulates apoptosis and autophagy of cardiomyocytes by targeting Mcl-1
}

\author{
YUHAI ZOU ${ }^{1,2}$, WENTING LIU ${ }^{3}$, JINXIA ZHANG ${ }^{2}$ and DINGCHENG XIANG ${ }^{1,2}$ \\ ${ }^{1}$ Southern Medical University, Guangzhou, Guangdong 510515; ${ }^{2}$ Department of Cardiology, \\ Guangzhou General Hospital of Guangzhou Military Command, Guangzhou, Guangdong 510010; \\ ${ }^{3}$ Department of ENT, Guangzhou First People's Hospital, Guangzhou, Guangdong 510180, P.R. China
}

Received July 9, 2015; Accepted May 5, 2016

DOI: $10.3892 / \mathrm{mmr} .2016 .5309$

\begin{abstract}
MicroRNAs (miRs) are a class of important regulators, which are involved in the regulation of apoptosis. Oxidative stress-induced apoptosis is the predominant factor accounting for cardiac ischemia-reperfusion injury. miR-153 has been previously shown to have an antitumor effect in cancer. However, whether miR-153 is involved in oxidative stress-induced apoptosis in the heart remains to be elucidated. To this end, the present study used reverse transcription-quantitative polymerase chain reaction to detect miR-153 levels upon oxidative stress, and evaluated apoptosis, autophagy and expression of critical genes by western blotting. A luciferase assay was also used to confirm the potential target gene. In the present study, it was found that the expression of miR-153 was significantly increased upon $\mathrm{H}_{2} \mathrm{O}_{2}$ stimulation, and the inhibition of endogenous miR-153 decreased apoptosis. To further identify the mechanism underlying the pro-apoptotic effect of miR-153, the present study analyzed the 3'untranslated region of myeloid cell leukemia-1 (Mcl-1), and found that Mcl-1 was potentially targeted by miR-153. The forced expression of miR-153 inhibited the expression of Mcl-1 and luciferase activity, which was reversed by its antisense inhibitor. Furthermore, it was shown that the inhibition of miR-153 induced autophagy during oxidative stress, and that its effects of autophagy induction and apoptosis inhibition were efficiently abrogated by Mcl-1 small interfering RNA. In conclusion, the results of the present study elucidated a novel mechanism by which miR-153 regulates the survival of cardimyocytes during oxidative stress through the modulation of apoptosis and autophagy. These effects may be mediated directly by targeting Mcl-1. These finding revealed the
\end{abstract}

Correspondence to: Professor Dingcheng Xiang, Department of Cardiology, Guangzhou General Hospital of Guangzhou Military Command, 111 Liuhua Road, Guangzhou, Guangdong 510010, P.R. China

E-mail: dcxiang2013@163.com

Key words: cardiomyocyte, autophagy, microRNA-153, apoptosis potential clinical value of miR-153 in the treatment of cardiovascular disease.

\section{Introduction}

The normal heart rhythm is particularly dependent upon proper contractile function of cardiomyocytes. However, the reactive oxygen species (ROS) generated under several pathological conditions, including ischemia-reperfusion injury, may be one of the major causes of heart muscle injury (1). ROS lead to an imbalanced intracellular redox condition, which is essential for the induction of apoptosis and impairment of ATP production. Due to the limited regenerative capacity of cardiomyocytes, understanding the mechanisms of oxidative stress-induced apoptosis may potentially assist in developing novel treatment or preventative strategies for ischemia-reperfusion injury of the heart.

Previous studies of microRNAs (miRs) have indicated a a number of novel mechanisms of heart diseases. It has been reported that miRs not only control arrhythmogenesis, but are also significantly involved in the regulation of cardiomyocyte death (2-4). Previously, miR-153 has been shown to suppress cancer cell proliferation, invasion and migration (5-8). Despite several targets, which have been identified in cancer cells, the role of miR-153 in cardiomyocytes remains to be fully elucidated.

As one of the major cascades of apoptosis, the intrinsic apoptotic pathway is initiated from mitochondria and is tightly controlled by B cell-lymphoma-2 (Bcl-2) family member proteins. One member of the Bcl-2 family, myeloid cell leukemia-1 (Mcl-1), has been reported to exert critical functions in apoptosis and mitochondrial homeostasis (9-13). Previous studies have demonstrated that the loss of Mcl-1 is essential in heart failure $(10,14)$. However, the mechanism by which Mcl-1 is regulated under oxidative stress remains to be fully elucidated. A mechanism revealed by a previous study showed that Mcl-1 functions to affect autophagy, a major degradation process in the lysosome, which is critical for cardiac homeostasis (10). A number of studies have shown that autophagy has a beneficial effect on the survival of cardiomyocyte under conditions of stress (15-17). However, whether Mcl-1 is involved in the regulation of pro-survival autophagy, which acts against oxidative damage, remains to be elucidated. 
The present study aimed to identify a novel microRNA-associated mechanism in cardiac oxidative stress. A potential binding site of miR-153 was identified in the Mcl-1 3'-untranslated region, suggesting that miR-153 may be involved in this process. Therefore, multiple experimental methods were used to confirm our hypothesis that miR-153 regulates cardiomyocytes survival upon oxidative stimuli. The present study identified a novel role of miR-153 in regulating apoptosis and autophagy, and thus may provide certain clinical implications for the treatment of oxidative stress associated heart syndrome.

\section{Materials and methods}

Cell culture. A primary culture of rat ventricular cardiomyocytes was used, as described previously (18). Each time, 10 neonatal Sprague-Dawley rats (within 2-days-old) were used for cell culture. The neonatal rats were purchased from the Experimental Animal Center of Southern Medical University (Guangzhou, China). Male and female rats used to breed the neonatal rats were housed at a constant temperature of $22^{\circ} \mathrm{C}$ with $50 \%$ humidity and $12 \mathrm{~h}$ light/dark cycles. The rats were sacrificed with $\mathrm{CO}_{2}$. Briefly, the hearts of neonatal rats were dissected and rinsed in cold phosphate-buffered saline (PBS), followed by being cut into several $2 \times 2 \mathrm{~mm}$ sections. The heart tissues were then digested with a series of $0.25 \%$ trypsin solution. Following the completion of digestion, the cell suspension was collected. To isolate the fibroblasts, the suspension was plated in flasks for $2 \mathrm{~h}$ at $37^{\circ} \mathrm{C}$. The cardiomyocytes were then diluted $(25,000$ cells $/ \mathrm{ml})$ and plated elsewhere. The cardiomyocytes were cultured in Dulbecco's modified Eagle's medium (GE Healthcare Life Sciences HyClone Laboratories, Logan, UT, USE) supplemented with $10 \%$ fetal bovine serum (Sigma-Aldrich, St. Louis, MO, USA) and penicillin-streptomycin solution at $37^{\circ} \mathrm{C} . \mathrm{H}_{2} \mathrm{O}_{2}$ was used at the final concentration of $100 \mu \mathrm{M}$. The present study was approved by the Ethics Committee of Southern Medical University (Guangzhou, China).

Transfection. A standard transfection procedure was used for delivering miR-153 or miR-153 antisense inhibitor into the cells. Lipofectamine 2000 tranfection reagent (Invitrogen; Thermo Fisher Scientific, Inc., Waltham, MA, USA) was used, according to the manufacturer's protocol for the kit. The Mcl-1 small interfering (si)RNA, miR-153 and miR-153 antisense inhibitor were designed and synthesized by Guangzhou RiboBio Co., Ltd. (Guangzhou, China). The final transfection concentrations for siRNA and miR were $150 \mathrm{nmol} / \mathrm{l}$.

MTT assay. To assess the extent of cell death, an MTT method was used. Briefly, the cells were plated $(25,000$ cells $/ \mathrm{ml})$ in 96-well plates and treated, as indicated in each experiment. The cells were treated with $100 \mu \mathrm{M} \mathrm{H}_{2} \mathrm{O}_{2}$ for 6,12 and $24 \mathrm{~h}$. For cells transfected with anti-miR-153 or anti-miR-153 in combination with si-Mcl-1, cells were treated with $100 \mu \mathrm{M} \mathrm{H}_{2} \mathrm{O}_{2}$ for $24 \mathrm{~h}$. Following treatment, $20 \mu \mathrm{l}$ MTT $(5 \mathrm{mg} / \mathrm{ml})$ solution was added to each well, and the cells were allowed to incubate for another $4 \mathrm{~h}$ at $37^{\circ} \mathrm{C}$. The culture medium was then discarded prior to visualization with dimethyl sulfoxide. The absorbance value at $490 \mathrm{~nm}$ was determined using a spectrophotometer (BioTek, Winooski, VT, USA).
Reverse transcription-quantitative polymerase chain reaction (RT-qPCR) analysis. Total RNA was isolated from the cardiomyocytes using TRIzol reagent (Invitrogen; Thermo Fisher Scientific, Inc.), according to the manufacturer's protocol. Reverse transcription was performed using a specific stem-loop primer obtained from Guangzhou RiboBio Co., Ltd. SYBR green master mix (Promega Corp., Madison, WI, USA) was used to amplify the cDNA $(1: 15 ; 5 \mu \mathrm{l})$ with specific primers for miR-153 (Guangzhou RiboBio Co., Ltd.) on an ABI7500 FAST Real-Time PCR system (Applied Biosystems; Thermo Fisher Scientific, Inc.). The following cycles were used: 40 cycles at $95^{\circ} \mathrm{C}$ for $2 \mathrm{~min}, 95^{\circ} \mathrm{C}$ for $30 \mathrm{sec}$ and $58^{\circ} \mathrm{C}$ for $40 \mathrm{sec}$. U6 was used as an internal control for normalization. The fold changes in miR-153 in each experimental group were calculated by normalizing to the negative control (NC) or control group using the $2^{-\Delta \Delta \mathrm{Cq}}$ method.

Western blot analysis. Western blot analysis was performed to detect the expression levels of the proteins of interest. Briefly, the cells were homogenized with SDS lysis buffer (Beyotime Institute of Biotechnology, Beijing, China) and denatured with sample buffer (Beyotime Institute of Biotechnology). The protein quantity was determined using a bicinchoninic acid assay. Electrophoresis of $30 \mu \mathrm{g}$ protein on an SDS-PAGE gel was performed, followed by transfer onto the supporting media of a polyvinylidene fluoride membrane. The membranes were blocked with $5 \%$ non-fat milk at room temperature for $2 \mathrm{~h}$. The proteins were identified by incubation with specific primary antibodies overnight at $4^{\circ} \mathrm{C}$, washed with PBS containing Tween-20 three times, followed by horseradish-peroxidase-conjugated secondary antibody specification for $40 \mathrm{~min}$ at room temperature. The membranes were washed as before. The bands were visualized using an ECL kit (Beyotime Institute of Biotechnology). Antibodies for LC3 (cat. no. 2775; 1:500), cleaved caspase-3 (cat. no. 9664; 1:500) and cleaved caspase-9 (cat. no. 9507; 1:1,000) were purchased from Cell Signaling Technology, Inc. (Danvers, MA, USA), antibodies for $\beta$-actin (cat. no. sc-130656; 1:1,000) and Mcl-1 (cat. n o. sc-819; 1:1,000), and secondary antibody was horseradis peroxidase-linked goat anti-rabbit immunoglobulin G (cat. no. sc-2301; 1:2,000) were purchased from Santa Cruz Biotechnology, Inc. (Santa Cruz, CA, USA). Protein bands were quantified using Quantity One software (v4.62; Bio-Rad Laboratories, Hercules, CA, USA).

Dual luciferase activity assay. A fragment of the Mcl-1 3'UTR was amplified by PCR, and the short sequence was inserted into the 3'UTR of an pMIRGLO construct, which expresses firefly luciferase. HEK293 cells $(20,000 / \mathrm{ml})$ were co-transfected with $0.1 \mu \mathrm{g}$ firefly luciferase reporter containing the Mcl-1 3'UTR and miR-153 or its antisense inhibitor, along with pRL-TK as an internal control using Lipofectamine 2000 (Thermo Fisher Scientific, Inc.). The 3'-UTR sequence was amplified from the cDNA using the primer: forward: 5'-GAGCTCTACAAGAGGGTGA AGGAGGA-3' and reverse: 5'-AAGCTTCAGGCTTAAA CTTGTGTTAAAC-3'. The amplification program was as follows: $95^{\circ} \mathrm{C}$ for $10 \mathrm{~min} ; 35$ cycles at $95^{\circ} \mathrm{C}$ for $30 \mathrm{sec}, 56^{\circ} \mathrm{C}$ for $30 \mathrm{sec}$ and $72^{\circ} \mathrm{C}$ for $45 \mathrm{sec}$; followed by $72^{\circ} \mathrm{C}$ for $5 \mathrm{~min}$. The empty pMIRGLO plasmid and the PCR products were 
A

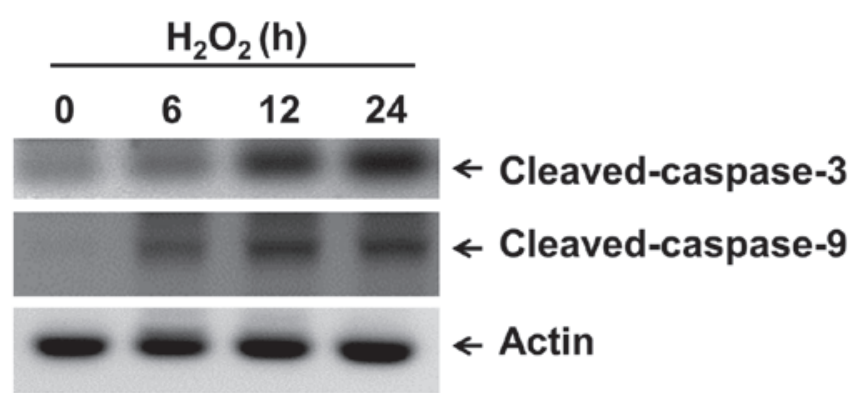

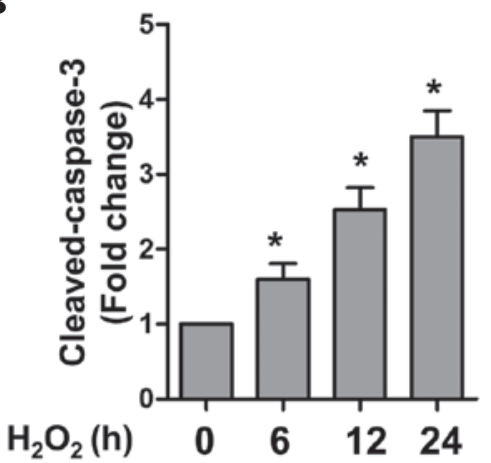

C

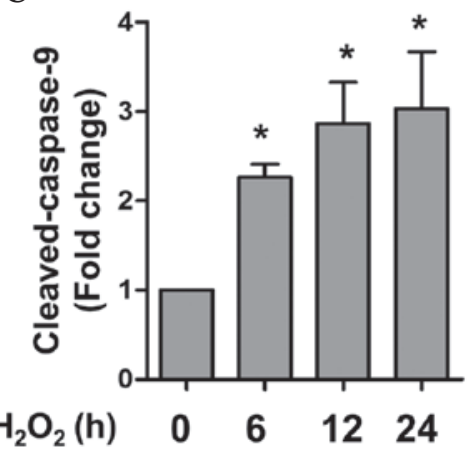

D

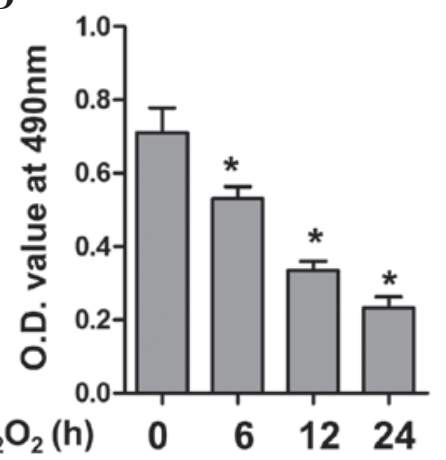

E

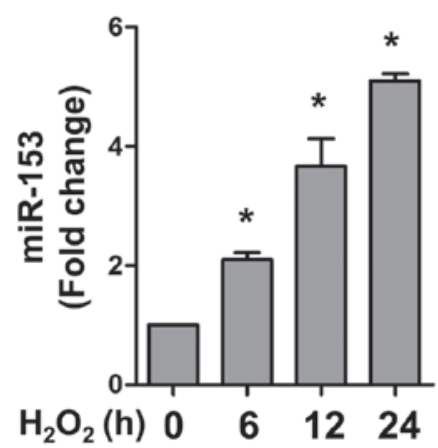

Figure 1. miR-153 is upregulated on exposure to apoptotic stimuli induced by oxidative stress. (A) Expression levels of cleaved-caspase-3 and cleaved-caspase-9 following $\mathrm{H}_{2} \mathrm{O}_{2}$ stimulation for various periods or time. Statistical analyses of the band densities of (B) cleaved caspase-3 and (C) cleaved caspase-9. (D) Cell viability of cardiomyocytes at each time point. (E) Relative expression of miR-153 at each time point. Data are presented as the mean \pm standard error of the mean. ${ }^{*} \mathrm{P}<0.05$, vs. control ( $\left.\mathrm{n}=5\right)$. miR, microRNA; O.D., optical density.

digested with HindIII and $\mathrm{SacI}$ at $37^{\circ} \mathrm{C}$ for $1 \mathrm{~h}$, followed by T4 ligation reaction at room temperature for $2 \mathrm{~h}$. The ligation product was then transformed into competent cells (Transgene Biotech, Beijing, China) and the positive plasmid was verified by PCR. All the restriction enzymes were purchase from Takara Bio., Inc. (Dalian, China). The luciferase activity in each group was normalized to that of the renilla luciferase expressed by the pRL-TK plasmid. The whole procedure was performed using a Dual-Luciferase Reporter Assay system (Promega Corp.) in accordance with the manufacturer's protocol.

Statistical analysis. All data are presented as the mean \pm standard error of the mean. Comparisons between two groups were performed using Student's $t$-test; comparisons between three groups were performed using one way analysis of variance followed by a Bonferroni test. SPSS 19.0 software (IBM SPSS, Chicago, IL, USA) was used for analysis. $\mathrm{P}<0.05$ (two-tailed) was considered to indicate a statistically significant difference.

\section{Results}

miR-153 is upregulated under apoptotic stimuli induced by oxidative stress. The present study first examined the expression level of miR-153 under oxidative stress. The cardiomyocytes were treated with $\mathrm{H}_{2} \mathrm{O}_{2}$ for different time periods to induce apoptosis in the cardiomyocytes. Using western blot analysis, $\mathrm{H}_{2} \mathrm{O}_{2}$ was found to induce caspase-3 and 9 activation in a time-dependent manner (Fig. 1A-C). Accordingly, a significant decrease in cell viability was observed under these conditions, as revealed by the MTT assay (Fig. 1D). RT-qPCR was used to detect the expression of miR-153 at various time points. In accordance with the expression of the apoptotic markers, caspase-3 and 9, miR-153 was also upregulated in a time-dependent manner. These results confirmed the successful establishment of an apoptotic model in the cardiomyocytes, and indicated that miR-153 may be involved in oxidative stress-induced apoptosis.

Inhibition of miR-153 reduces the apoptosis of cardiomyocytes. Following the above observation that miR-153 was upregulated during oxidative stress, the present study subsequently transfected cells with the antisense inhibitor of miR-153 (anti-miR-153) to manipulate its endogenous level. Anti-miR-153 significantly reduced the expression of miR-153 under the $\mathrm{H}_{2} \mathrm{O}_{2}$ stimuli (Fig. 2A). The results of the western blot analysis showed that the expression levels of activated caspase-3 and caspase-9 were significantly lower following miR-153 inhibition (Fig. 2B-D). Consistently, the inhibition of miR-153 by its antisense inhibitor increased the survival of cardiomyocytes upon $\mathrm{H}_{2} \mathrm{O}_{2}$ stimulation (Fig. 2E).

Mcl-1 is a target of miR-153. miRs commonly function to repress gene expression by binding to the 3 'UTR region of 
A

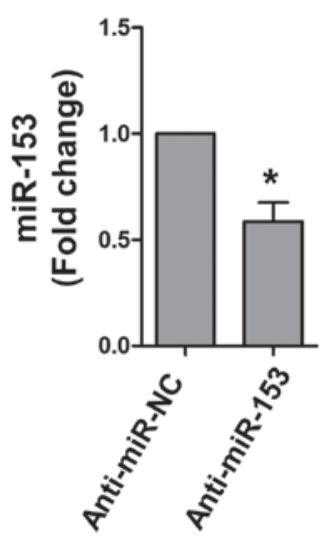

C

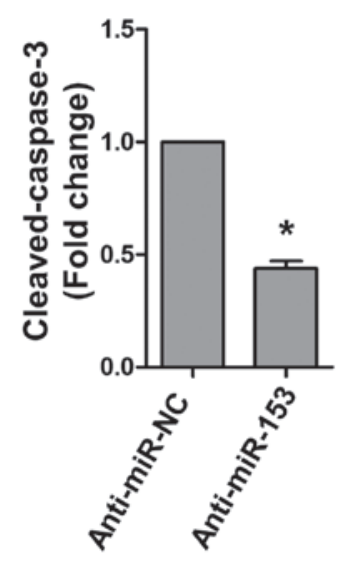

B

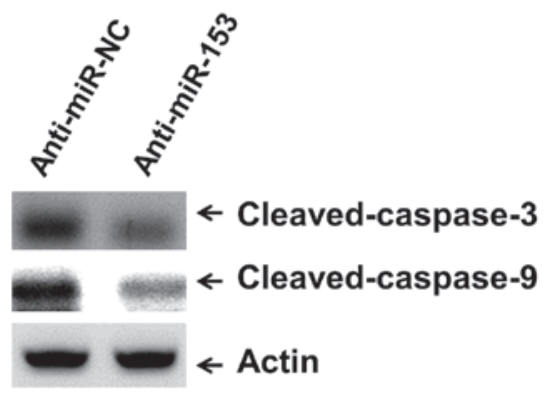

D

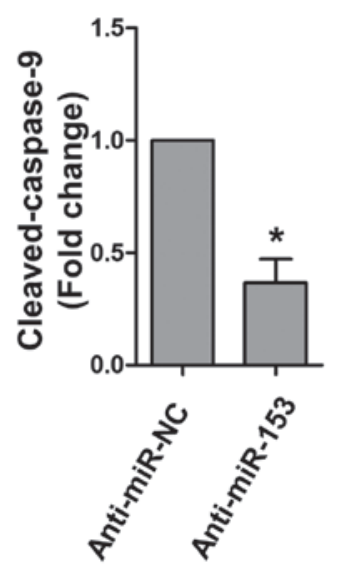

$\mathbf{E}$

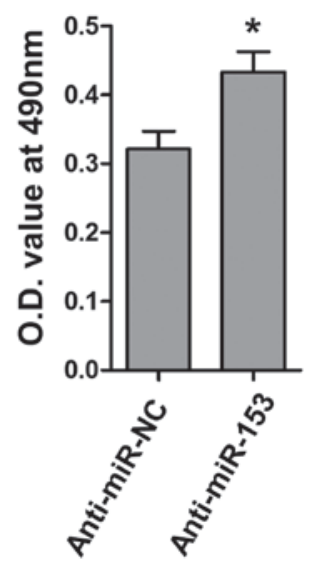

Figure 2. Inhibition of miR-153 reduces apoptosis of cardiomyocytes. (A) Relative expression of miR-153 following anti-miR-153 transfection. (B) Expression levels of cleaved-caspase-3 and cleaved-caspase-9 in cardiomyocytes subjected to different treatments. Statistical analyses of the band densities of (C) cleaved capsase-3 and (D) cleaved caspase-9. Cardiomyocytes were transfected with anti-miR-153 or anti-miR-NC for 48 h, and were then incubated with $\mathrm{H}_{2} \mathrm{O}_{2}$ for 24 h. (E) Cell viability of the transfected cardiomyocytes. Data are presented as the mean \pm standard error of the mean. * $\mathrm{P}<0.05$, vs. anti-miR-NC ( $=5$ ). miR, microRNA; anti-miR-53, miR-153 antisense inhibitor; NC, negative control; O.D., optical density.

A

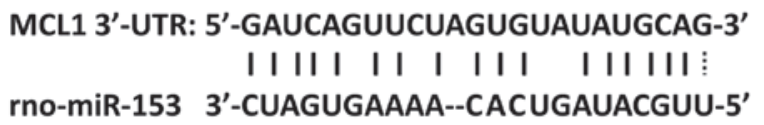

B

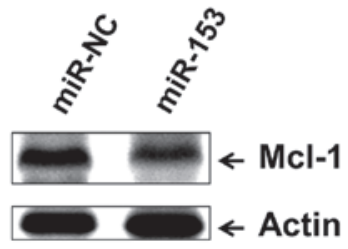

C

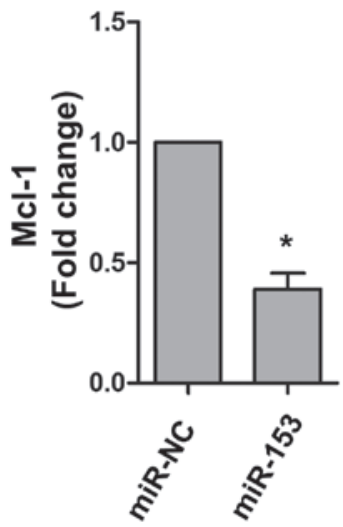

D

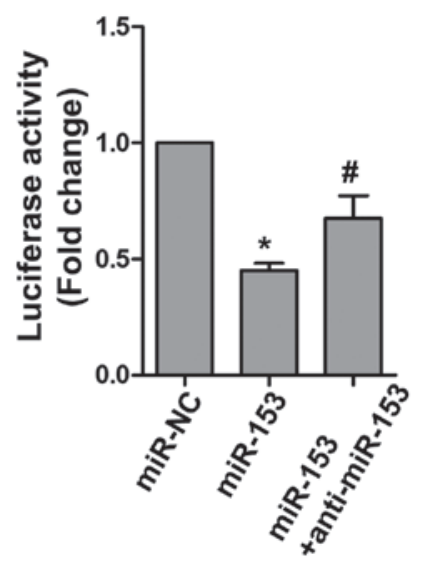

Figure 3. Mcl-1 is a target of miR-153. (A) Predicted binding sites of rno- miR-153 and rat Mcl-1 3'UTR. (B) Enforced expression of miR-153 inhibited the expression of Mcl-1. (C) Statistical analysis of the band densities of Mcl-1. (D) miR-153 inhibited luciferase activity, whereas anti-miR-153 partially reversed this inhibition. Data are presented as the mean \pm standard error of the mean. ${ }^{~} \mathrm{P}<0.05$, vs. miR-NC; ${ }^{*} \mathrm{P}<0.05$, vs. miR-153 (n=5). miR, microRNA; ${ }^{\prime} \mathrm{UTR}$, 3' untranslated region; Mcl-1, myeloid cell leukemia-1; NC, negative control. 
A

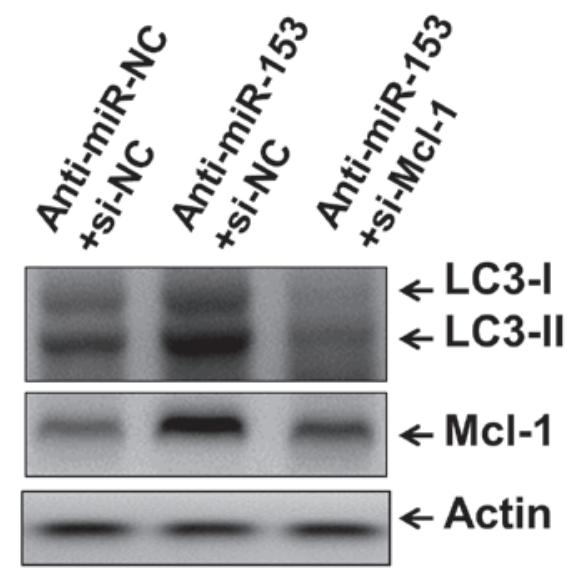

C

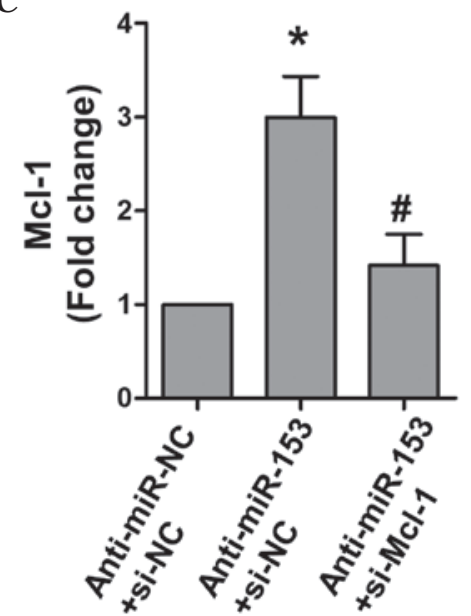

B

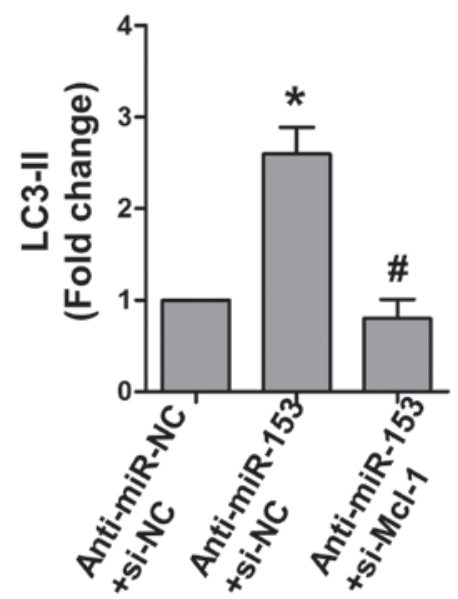

D

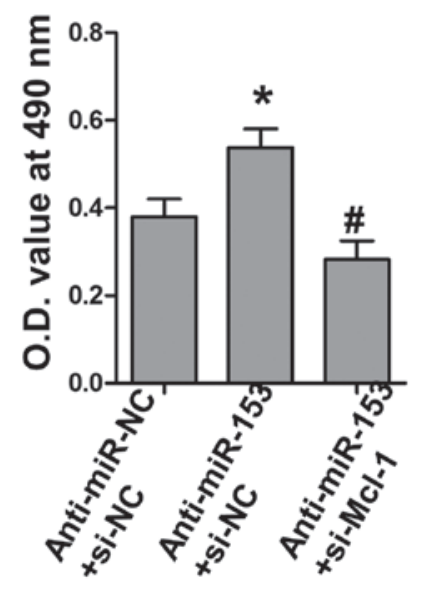

Figure 4. miR-153 inhibits Mcl-1-dependent autophagy. (A) Expression levels of LC3 and Mcl-1 following transfection with anti-miR-153 or si-Mcl-1. Statistical analyses of band densities of (B) LC3-II and (C) Mcl-1. The cardiomyocytes were co-trasnfected with anti-miR-153 and si-Mcl-1 or their negative control strands for $48 \mathrm{~h}$, as indicated, and were then incubated with $\mathrm{H}_{2} \mathrm{O}_{2}$ for $24 \mathrm{~h}$. (D) Cell viability of the transfected cardiomyocytes. Data are presented as the mean \pm standard error of the mean. "P<0.05, vs. anti-miR-NC+si-NC; ${ }^{*} \mathrm{P}<0.05$, vs. anti-miR-153+si-NC ( $=5$ ). miR, microRNA; Mcl-1, myeloid cell leukemia-1; si, small interfering; NC, negative control; O.D., optical density.

mRNA. Using bioinformatical alignment, the present study identified a potential binding site within the 3'UTR of Mcl-1 (Fig. 3A). Whether this predicted region is actually targeted by miR-153 was then assessed. The cardiomyocytes were transfected with miR-153, and it was found that the expression of Mcl-1 was significantly inhibited, compared with the NC group of cells (Fig. 3B and C). A luciferase assay was also used to examine the association between Mcl-1 and miR-153. As shown in Fig. 3D, the overexpression of miR-153 markedly inhibited luciferase activity, and anti-miR-153 rescued the inhibitory effect of miR-153 on luciferase activity.

miR-153 inhibits Mcl-1-dependent autophagy. As Mcl-1 has been established as a target of miR-153, and it has been previously shown to affect cardiac autophagy, the present study investigated whether Mcl-1-dependent autophagy is also under the control of miR-153 $(10,19)$. The inhibition of miR-153 under $\mathrm{H}_{2} \mathrm{O}_{2}$ stimulation markedly increased the expression of the autophagy marker, LC3-II, whereas the inhibition of Mcl-1 inhibited this effect (Fig. 4A-C). In particular, the MTT assay showed that the inhibition of Mcl-1 inhibited the protective effect of anti-miR-153, whereas inhibiting anti-miR-153 induced autophagy, suggesting that Mcl-1-dependent autophagy was critically involved in the promotion of cell death by miR-153 (Fig. 4D).

\section{Discussion}

In the present study, it was demonstrated that miR-153 was involved in oxidative stress-induced cell death in cardiomyocytes. On investigating the molecular mechanism by which miR-153 regulated cell survival, the present study identified Mcl-1 as its direct target. As a consequence, the cardiomyocytes exhibited limited autophagic activity and enhanced apoptosis, which may have accounted, at least partially, for the $\mathrm{H}_{2} \mathrm{O}_{2}$-induced cardiomyocyte death. These results may have important clinical implications for the prevention and treatment of cardiac syndromes, including ischemia-reperfusion injury.

miRs have emerged as a class of important regulators in the heart. The interplay between miRs and several critical regulators in the apoptotic machinery have attracted wide attention in cardiac investigations. For example, a previous study by Wang et al showed that miR-361 inhibits apoptosis by regulating 
prohibitin during myocardial ischemia (20), and Xu et al reported that the $\beta$-blocker, carvedilol, exerts a protective function on cardiomyocytes against oxidative stress-induced apoptosis by inducing the expression of miR-133 (21). These earlier findings suggest that miRs may have a profound effect on cardimyocyte survival. miR-153 is located on chromosome 6 in the rat genome, and previous studies have reported that miR-153 inhibits the growth and invasive behavior of cancer cells. Bai et al reported its importance in pancreatic ductal adenocarcinoma (22), and Zhou et al showed its antitumor effect in ovary cancer cells (6). However, there are few reports on the direct role of miR-153 on apoptosis, particularly in heart cells. The present study provided the first evidence, to the best of our knowledge, that miR-153 was increased upon apoptosis induced by $\mathrm{H}_{2} \mathrm{O}_{2}$ in cardiomyocytes, and that modulation in its level had a direct effect on cell apoptosis.

Apoptosis and autophagy are two essential biological processes, which act reciprocally to control cell survival under oxidative stress. It has been reported that basal autophagic activity is required for heart cells to maintain homeostasis, and that inhibition of this process by the deletion of its essential gene, ATG5, leads to heart failure (23). It has also been demonstrated that autophagy is required for cardimyocytes to confer their anti-apooptotic effects (24). Previous studies have shown that Mcl-1 is involved, not only in the apoptotic machinery, but also in the quality control of mitochondria by autophagy. Thomas et al reported that the deletion of Mcl-1 in heart muscle results in impaired autophagy and heart failure (10). The present study identified a free-energy favorable binding site of miR-153 within the Mcl-1 3'UTR, suggesting that miR-153 may modulate cell survival through the downstream effect of Mcl-1. Following validation of Mcl-1 as a direct target of miR-153, the present study investigated whether autophagy was also involved. Consistent with previous studies, the increased expression of the autophagic marker, LC3-II, was observed upon miR-153 inhibition, which was reversed by Mcl-1 silencing. Thus, the present study is the first, to the best of our knowledge, to provide a novel mechanism underlying the regulation of cell apoptosis and autophagy in heart cells by miR-153.

The way in which miRs are regulated under different pathological conditions is an important issue and requires further understanding of the behavior of cardiomyocytes. Previous studies demonstrating the regulation of transcriptional factors of miRs may provide insights into this issue. For example, serum response factor transcriptionally regulates miR-1 in the heart, and p53 transcriptionally downregulates the expression of miR-499 to promote apoptosis $(25,26)$. It is conceivable that several oxidative stress-specific transcriptional factors may regulate the expression of miR-153. Further investigation is warranted to investigate the upstream events of miR-153.

In conclusion, the present study demonstrated the key role of miR-153 in oxidative stress-induced apoptosis. Mcl-1 was identified as a novel target, through which miR-153 regulated essential programs, including apoptosis and autophagy. These results may provide novel clues for understanding the control by miRs of multiple effects under certain stresses. The results of the present study may also guide the development of novel therapies for cardiac syndromes.

\section{References}

1. Hafstad AD, Nabeebaccus AA and Shah AM: Novel aspects of ROS signalling in heart failure. Basic Res Cardiol 108: 359, 2013.

2. Wang J and Martin JF: Macro advances in microRNAs and myocardial regeneration. Curr Opin Cardiol 29: 207-213, 2014.

3. Sala V, Bergerone S, Gatti S, Gallo S, Ponzetto A, Ponzetto C and Crepaldi T: MicroRNAs in myocardial ischemia: Identifying new targets and tools for treating heart disease. New frontiers for miR-medicine. Cell Mol Life Sci 71: 1439-1452, 2014.

4. Port JD and Sucharov C: Role of microRNAs in cardiovascular disease: Therapeutic challenges and potentials. J Cardiovasc Pharmacol 56: 444-453, 2010.

5. Xia W, Ma X, Li X, Dong H, Yi J, Zeng W and Yang Z: miR-153 inhibits epithelial-to-mesenchymal transition in hepatocellular carcinoma by targeting Snail. Oncol Rep 34: 655-662, 2015.

6. Zhou J, Xie M, Shi Y, Luo B, Gong G, Li J, Wang J, Zhao W, Zi Y, Wu X and Wen J: MicroRNA-153 functions as a tumor suppressor by targeting SET7 and ZEB2 in ovarian cancer cells. Oncol Rep 34: 111-120, 2015.

7. Hua HW, Jiang F, Huang Q, Liao Z and Ding G: MicroRNA-153 promotes $\mathrm{Wnt} / \beta$-catenin activation in hepatocellular carcinoma through suppression of WWOX. Oncotarget 6: 3840-3847, 2015.

8. Shan N, Shen L, Wang J, He D and Duan C: MiR-153 inhibits migration and invasion of human non-small-cell lung cancer by targeting ADAM19. Biochem Biophys Res Commun 456: 385-391, 2015.

9. Carroll RG, Hollville E and Martin SJ: Parkin sensitizes toward apoptosis induced by mitochondrial depolarization through promoting degradation of Mcl-1. Cell Rep 9: 1538-1553, 2014.

10. Thomas RL, Roberts DJ, Kubli DA, Lee Y, Quinsay MN, Owens JB, Fischer KM, Sussman MA, Miyamoto S and Gustafsson ÅB: Loss of MCL-1 leads to impaired autophagy and rapid development of heart failure. Genes Dev 27: 1365-1377, 2013.

11. Jokinen E and Koivunen JP: Bcl-xl and Mcl-1 are the major determinants of the apoptotic response to dual PI3K and MEK blockage. Int J Oncol 47: 1103-1110, 2015.

12. Cerella C, Muller F, Gaigneaux A, Radogna F, Viry E, Chateauvieux S, Dicato M and Diederich M: Early downregulation of Mcl-1 regulates apoptosis triggered by cardiac glycoside UNBS1450. Cell Death Dis 6: e1782, 2015.

13. Varadarajan S, Poornima P, Milani M, Gowda K, Amin S, Wang HG and Cohen GM: Maritoclax and dinaciclib inhibit MCL-1 activity and induce apoptosis in both a MCL-1-dependent and -independent manner. Oncotarget 6: 12668-12681, 2015.

14. Wang X, Bathina M, Lynch J, Koss B, Calabrese C, Frase S, Schuetz JD, Rehg JE and Opferman JT: Deletion of MCL-1 causes lethal cardiac failure and mitochondrial dysfunction. Genes Dev 27: 1351-1364, 2013.

15. Li L, Xu J, He L, Peng L, Zhong Q, Chen L and Jiang Z: The role of autophagy in cardiac hypertrophy. Acta Biochim Biophys Sin, 2016

16. Jia Z, Liu Y, Su H, Li M, Zhang M, Zhu Y, Li T, Fang Y and Liang S: Safflower extract inhibiting apoptosis by inducing autophagy in myocardium derived H9C2 cell. Int J Clin Exp Med 8: 20254-20262, 2015.

17. Schiattarella GG and Hill JA: Therapeutic targeting of autophagy in cardiovascular disease. J Mol Cell Cardiol, 2015.

18. Wang K, Liu F, Zhou LY, Ding SL, Long B, Liu CY, Sun T, Fan YY, Sun L and Li PF: miR-874 regulates myocardial necrosis by targeting caspase-8. Cell Death Dis 4: e709, 2013.

19. Xu J, Liao X and Wong C. Downregulations of B-cell lymphoma 2 and myeloid cell leukemia sequence 1 by microRNA 153 induce apoptosis in a glioblastoma cell line DBTRG-05MG. Int J Cancer 126: 1029-1035, 2010.

20. Wang K, Liu CY, Zhang XJ, Feng C, Zhou LY, Zhao Y and Li PF: miR-361-regulated prohibitin inhibits mitochondrial fission and apoptosis and protects heart from ischemia injury. Cell Death Differ 22: 1058-1068, 2015.

21. Xu C, Hu Y, Hou L, Ju J, Li X, Du N, Guan X, Liu Z, Zhang T, Qin W, et al: $\beta$-Blocker carvedilol protects cardiomyocytes against oxidative stress-induced apoptosis by up-regulating miR-133 expression. J Mol Cell Cardiol 75: 111-121, 2014.

22. Bai Z, Sun J, Wang X, Wang H,Pei H and Zhang Z: MicroRNA-153 is a prognostic marker and inhibits cell migration and invasion by targeting SNAI1 in human pancreatic ductal adenocarcinoma. Oncol Rep 34: 595-602, 2015. 
23. Nakai A, Yamaguchi O, Takeda T, Higuchi Y, Hikoso S, Taniike M, Omiya S, Mizote I, Matsumura Y, Asahi M, et al The role of autophagy in cardiomyocytes in the basal state and in response to hemodynamic stress. Nat Med 13: 619-624, 2007.

24. Dutta D, Xu J, Kim JS, Dunn WA Jr and Leeuwenburgh C: Upregulated autophagy protects cardiomyocytes from oxidative stress-induced toxicity. Autophagy 9: 328-344, 2013.
25. Wang JX, Jiao JQ, Li Q, Long B, Wang K, Liu JP, Li YR and Li PF: miR-499 regulates mitochondrial dynamics by targeting calcineurin and dynamin-related protein-1. Nat Med 17: 71-78, 2011.

26. Zhao Y, Samal E and Srivastava D: Serum response factor regulates a muscle-specific microRNA that targets Hand2 during cardiogenesis. Nature 436: 214-220, 2005. 